\title{
Ters Sarkaç Sistemi İçin LQR Kontrolcü Tasarımında Genetik Algoritma Optimizasyonu
}

\author{
Mehmet Tayyip Özdemir ${ }^{1}$, Muhammet Mevlüt Karaca ${ }^{2}$, Ali Tahir Karaşahin ${ }^{3 *}$ \\ ${ }^{1}$ Karabük Üniversitesi, Mühendislik Fakültesi, Makine Mühendisliği Bölümü, Karabük, Türkiye (ORCID: 0000-0002-4290-0045) \\ ${ }^{2}$ Karabük Üniversitesi, Mühendislik Fakültesi, Makine Mühendisliği Bölümü, Karabük, Türkiye (ORCID: 0000-0001-9644-3663) \\ ${ }^{3}$ Karabük Üniversitesi, Mühendislik Fakültesi, Mekatronik Mühendisliği Bölümü, Karabük, Türkiye (ORCID: 0000-0002-7440-1312)
}

(International Symposium on Multidisciplinary Studies and Innovative Technologies (ISMSIT) 2020 - 22-24 Ekim 2020)

(DOI: 10.31590/ejosat.820337)

ATIF/REFERENCE: Özdemir, M.T, Karaca, M.M., \& Karaşahin, A.T. (2020). Ters Sarkaç Sistemi İçin LQR Kontrolcü Tasarımında Genetik Algoritma Optimizasyonu. Avrupa Bilim ve Teknoloji Dergisi, (Özel Sayı), 163-171.

$\ddot{O} \mathbf{z}$

$\mathrm{Bu}$ çalışmada kontrol tekniklerinin performanslarını incelemek için sıklıkla tercih edilen ters sarkaç sistemi ele alınmıştır. Ters sarkaç sisteminin doğrusal olmayan yapısı nedeniyle de kontrolü zor bir mühendislik problemidir. Ters sarkaç problemine yönelik sistemin hareket denklemleri çıkartılmış, durum-uzay formunda ifade edilmiş ve tasarım kriterleri belirlenmiştir. Ters sarkaç sisteminde tasarım kriteri olarak arabanın ve sarkacın pozisyonlarını kontrol etmek hedeflenmiştir. Bu hedeflere uygun olarak kontrol tekniği belirlenmiştir. Kontrolcü olarak Lineer Kuadratik Regülatör (LQR) tekniği kullanılmıştır. LQR kontrolcüsü ile birden fazla durum değişkenleri kontrol edilebildiği için ters sarkaç sisteminde tercih edilmiştir. Gerçekleştirilen çalışmada LQR kontrolcüsünün performansını doğrudan etkileyen Q ve R matrisleri Genetik Algoritma ile optimize edilmiş̧ir. Optimize edilmiş LQR kontrolcüsü ve standart LQR kontrolcüsü olarak iki farklı yöntem uygulanmıştır. Genetik Algoritma geniş arama algoritmalarının aksine en iyiye ulaşmak için bir yaklaşımı olmadığından en iyiye ulaşamayabilir fakat zaman kısıtlamalarını dikkate almada en iyi algoritmalardan birisi olduğu için tercih edilmiştir. Ters sarkaç sisteminde yapılan optimizasyonlarda amaç fonksiyonları genellikle referans değere yükselme süresi, oturma süresi ve kalıcı durum hatalarının toplanması olarak kullanılmaktadır. Gerçekleştirilen çalışmada farklı olarak Genetik Algoritmanın uygunluk fonksiyonu için bir öneri sunulmuştur. Bu öneri, arabanın referans pozisyon değeri ile arabanın pozisyon değeri arasındaki farkın minimize edilmesi şeklinde tasarlanmıştır. Genetik Algoritma (GA) uygunluk fonksiyonunun çalışmada önerilen formül ile kullanıldığında kabul edilebilir sonuçlar ürettiği gösterilmiştir. Gerçekleştirilen deneyler sonucunda Genetik Algoritma ile optimizasyonu yapılan LQR kontrolcüsü, deneme yanılma yöntemiyle bulunan değerler ile çalışan $L Q R$ kontrolcüsüne göre daha başarılı olduğu gözlemlenmiştir. Aynı zamanda Q ve R matrisleri Genetik Algoritma ile belirlendiği için bu katsayıların belirlenmesinde kaybedilen zamanın önüne geçilmiştir.

Anahtar Kelimeler: Genetik Algoritma, LQR Kontrol, Optimizasyon, Ters Sarkaç.

\section{Genetic Algorithm Optimization in LQR Controller Design for Inverted Pendulum System}

\begin{abstract}
In this study, the frequently preferred inverted pendulum system is handled to examine the performance of control techniques. It is a difficult engineering problem to control due to the nonlinear nature of the inverted pendulum system. The motion equations of the system for the inverted pendulum problem were extracted, expressed in state-space form, and design criteria were determined. In the inverted pendulum system, it is aimed to control the positions of the cart and the pendulum as a design criterion. The Control technique has been determined by these goals. Linear Quadratic Regulator (LQR) technique is used as the controller. Since multiple state variables can be controlled with the LQR controller, it was preferred in the inverted pendulum system. The Q and R matrices that directly affect the performance of the LQR controller in the work carried out were optimized with the Genetic Algorithm. Two
\end{abstract}

\footnotetext{
* Sorumlu Yazar: Karabük Üniversitesi, Mühendislik Fakültesi, Mekatronik Mühendisliği Bölümü, Karabük, Türkiye, ORCID: 0000-0002-7440- 
different methods were applied as an optimized LQR controller and standard LQR controller. Unlike the wide search algorithms, the Genetic Algorithm may not reach the best because it does not have an approach to reach the best, but it was preferred because it is one of the best algorithms to consider time constraints. In the optimizations made in the inverted pendulum system, the fitness functions are generally used as the rise time to the reference value, the settling time, and the summation of the steady-state errors. In the study, a different proposal was presented for the fitness function of the Genetic Algorithm. This proposal is designed to minimize the difference between the car's reference position value and the car's current position value. The Genetic Algorithm fitness function has been shown to produce acceptable results when used with the formula suggested in the study. As a result of the experiments carried out, it was observed that the LQR controller, which was optimized with the Genetic Algorithm, was more successful than the LQR controller working with the values found by the trial and error method. At the same time, since the $\mathrm{Q}$ and $\mathrm{R}$ matrices are determined by the Genetic Algorithm, the time lost in determining these coefficients has been prevented.

Keywords: Genetic Algorithm, Inverted Pendulum, LQR Control, Optimization.

\section{Giriş}

Ters sarkaç sistemi kararsız ve doğrusal olmayan bir yapıya sahip olduğun için, çok sayıdaki kontrol metotlarının uygulanmasının ve doğrulanmasının test edilebileceği önemli bir kontrol mekanizmasıdır(Bilgiç vd., 2014). Bu sistemde kontrolün ana amacı, sarkacı dik konumda sabitlemektir. Bu işlem genellikle sarkaç çubuğunun takılı olduğu nesneyi(araba) hareket ettirerek gerçekleştirilmektedir. Bu nedenle, araba konumu ikincil bir değişken parametredir(Bakarac vd., 2018). Ters sarkaç sistemi, robot kollarındaki tekli mafsalların kontrolü, yürüyen robotların denge kontrolü gibi birçok yeni teknolojinin temelini oluşturmaktadır(Yeung \& Chen, 1990).

Ters sarkaç sistemiyle ilgili geçmişten günümüze kadar çok sayıda çalışmalar mevcut olup halen bu konu üzerinde çalışmalar devam etmektedir. Anderson 1989 yılında yapmış olduğu çalışmada, Ters sarkaç mekanizmasında sinir ağları uygulamasının diğer uygulamalarının aksine, performans geri beslemesinin her adımda kullanılamadığı ve yalnızca sarkaç düştüğünde veya yatay bir yolun sınırlarına ulaştığında bir başarısızlık sinyali olarak göründüğünü varsaymıştır. Bu problemi çözmek için, kontrolör gecikmiş performans değerlendirme, belirsizlik altında öğrenme ve doğrusal olmayan fonksiyonların öğrenilmesi konularını ele almıştır. Kararsız koşullardan kaçınmak ve sarkacı dengelemek için bu konuları ele alan pekiştirme ve zamansal fark öğrenme yöntemlerini sunmuştur(Anderson, 1989).

Mahfouz ve arkadaşları yaptığı çalışmada, matematiksel modelleri türetmek, karşılık gelen Simulink modelleri oluşturmak ve özellikle bir mekatroniği tasarlamak, inşa etmek ve kontrol etmek için mekatronik hareket kontrol uygulamalarında kullanılan elektrik DC motor sisteminin dinamik analizini yapmak için kullanılan farklı yaklaşımları ele almışlardır. Tek serbestlik derecesine sahip robot kolunu MATLAB/Simulink kullanarak doğrulamıştır(Mahfouz vd., 2013). Housner 1963 yılında yapmış olduğu çalışmada uzun ve ince binaların depreme karşı kalın binalara göre daha dayanıklı olduğunu görmüştür. Bu sebeple ters sarkaç tipi binaların deprem esnasında davranışını gözlemlemiştir. Binaları blok olarak modelleyip sabit, yatay bir ivme, tek bir sinüs darbesi ve bir deprem tipi uyarma ile devrilmesini analiz etmiştir(Housner, 1963).

Wang yapmış oluğu çalışmada, üç tipteki ters sarkacı kararlı hale getirmek ve davranışlarını gözlemlemek için PID denetleyicisi tasarlamıştır. Üç tür ters sarkaç arasındaki ilişskileri, Lagrange denklem modelleri ve durum denklem modelleri açısından analiz etmiştir. PID kontrolörleri ile üç tip ters sarkacın stabilizasyonu ve izleme kontrolünü gerçekleştirmiştir. Kontrol şeması sadece iyi bir dinamik performansa sahip olmakla kalmamış, aynı zamanda dıştaki büyük ve hızlı tepkilere karşı dayanıklılığa da sahip olmuştur(Wang, 2011). Okubanjo ve Oyetola 2019 yılında yaptıkları çalışmada, ters çevrilmiş bir sarkacın sistem dinamiklerini açıkça modelleyerek ve yatay konumda arabaya uygulanan giriş kuvvetini kontrol ederek sarkacı dikey konumda stabilize edecek farklı kontrol algoritmaları uygulamışlardır. Matematiksel model Lagrange yaklaşımının enerji özelliğine dayalı olarak türeterek ve kontrol algoritmaları MATLAB/Simulink ortamında türeterek matematiksel model üzerinde genişletmişlerdir. Bu nedenle, doğrusallaştırılmış ters sarkaç sisteminin kontrolü için Oransal-İntegral-Türev denetleyicisi (PID), Kutup Yerleştirmeli Geri Besleme denetleyicisi (PPFC), Lineer Karesel Regülatör denetleyicisi (LQR) ve Tahminsel (LQR + Tahminci) Doğrusal Karesel Regülatör denetleyicisi olmak üzere dört farklı kontrol algoritması önermişlerdir. Bu çalışma, önerilen kontrol algoritmalarını sistem yanıtı ve performansı açısından karşılaştırmaktadır(Okubanjo \& Oyetola, 2019).

Mevcut çalışmanın temel amacı, serbest konumda olan bir sarkacı yukarı sallamak ve sarkacı dik pozisyonda dengelemek ve sarkaç arabasını en kısa sürede sarkaç rayında belirli bir konuma taşımayı hedeflemişlerdir. Farklı kontrol stratejilerini deneylerle karşılaştırmışlar ve test etmişlerdir. Bulanık salınım algoritmasının enerji tabanlı salınım stratejileriyle karşılaştırılmışlar, doğrusal olmayan gerçek zamanlı uygulamalarda bulanık kontrol teorisini kullanmanın avantajlarını göstermişlerdir(Muskinja \& Tovornik, 2006). Razzaghi ve Jalali, ters sarkaç sistemini kontrol etmek için PID kontrolörlerini kullanan iki yöntem önermişlerdir. Birinci yöntem, sarkaç açısı altındaki araba pozisyonunun kontrolü, ikincisi ise araba pozisyonu altındaki sarkaç açısının kontrolüdür. Bu iki yöntemi birbiriyle karşılaştırarak, ikinci yöntemin daha iyi sonuçlar verdiği gösterilmiştir(Razzaghi \& Jalali, 2012). Shehu ve diğerleri bir ters sarkacın kontrolü için önerilen LQR, iki PID ve kutup yerleştirme kontrol yöntemi ve gerçekleştirdikleri sistem kontrolünün simülasyon sonuçlarını bu üç yöntemle karşılaştırmışlardır. Harici bozucu etki olması veya olmaması durumunda LQR kontrolörü ile en iyi performansı elde edilmiştir(Shehu vd., 2015). Prasad ve arkadaşları ters sarkaç sisteminin kontrolünü PID ve LQR kontrolörleri ile gerçekleştirmiş ve PID + LQR kontrol yönteminin PID kontrol yönteminden daha iyi sonuçlar verdiğini göstermiştir (Prasad vd., 2014). Ghanbari ve Farrokhi, çift eksenli ters sarkaç kontrolüne yeni bir yaklaşım getirmiştir. İlk olarak, iki eksenli ters sarkaç sistemini, merkezi olmayan kontrol teorisi yardımıyla iki ayrı alt sisteme bölünmüştür. Daha sonra, kayan mod yöntemini kontrol etmek için bir ayırma yöntemi kullanarak her bir alt sistemi iki yüzeye ayırırlar. Kayma modlu kontrolörler ayrıca iki bulanık sinir ağını öğrenmek için de kullanılmaktadır(Ghanbari \& Farrokhi, 2006). 


\section{Materyal ve Metot}

Ters sarkaç sistemine uygulanan $F$ kuvveti arabanın $x$ ekseninde pozisyon değişimine sebep olurken sarkaç kısmında ise $\theta$ açısının değişimine etki etmektedir. Öncelikle ters sarkaç sisteminin dinamik hareket denklemleri çıkartılacaktır. Dinamik hareket denklemleri belirli yaklaşımlarla doğrusallaştırıldıktan sonra kontrolcü tasarımına geçilecektir. Lineer sistemler için maliyet fonksiyonu tabanlı çalışan LQR kontrolcüsü ile ters sarkaç sistemi kontrol edilecektir. Tasarım kriterlerinden birisi arabanın referans konuma gönderilmesidir. Diğer tasarım kriteri ise arabanın referans takibi esnasında sarkacın açısının dengelenmesidir. LQR kontrolcü kazanç matrisleri GA ile optimize edilecektir. Kontrolcü tasarımı sonucunda standart ve GA ile optimize edilmiş LQR kontrolcüsünün performansları karşılaştırılacaktır.

\subsection{Matematiksel Modelleme}

Araba üzerinde konumlanmış ters sarkaç sistemi $(M)$ kütleli araba ile serbest dönme hareketi yapabilen $(m)$ kütleli ve $(l)$ boyundaki bir sarkaçtan oluşmaktadır.

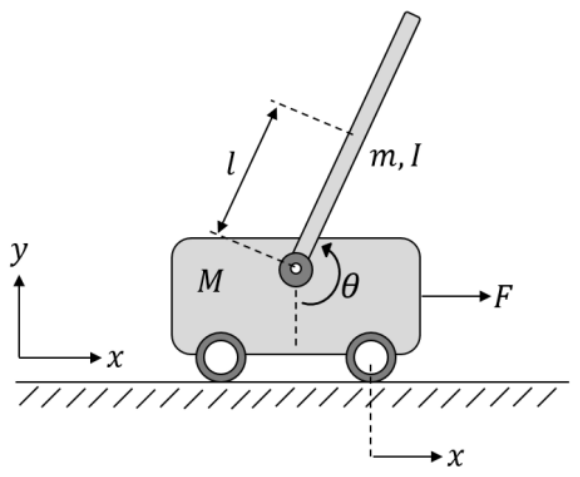

Şekil 1. Araball ters sarkaç modeli

Araba üzerinde konumlanmış sarkacın iki denge noktası mevcuttur. Bunlardan biri $\theta$ açısının sıfır olduğu kararsız denge noktasıdır. Diğeri ise $\pi$ olduğu kararlı denge noktasıdır. Bu çalışmadaki amaç ise sarkacın y ekseni ile yaptığı $\theta$ açısını sıfır olarak dengede tutmaktır. $\mathrm{Bu}$ amacı gerçekleştirmek için arabaya $\mathrm{x}$ ekseninde $\mathrm{F}$ girişi kullanılacaktır. Çıkışlar ise sarkaç açısı olan $\theta$ ve arabanın konumu olan $\mathrm{x}$ durum değişkenleridir.

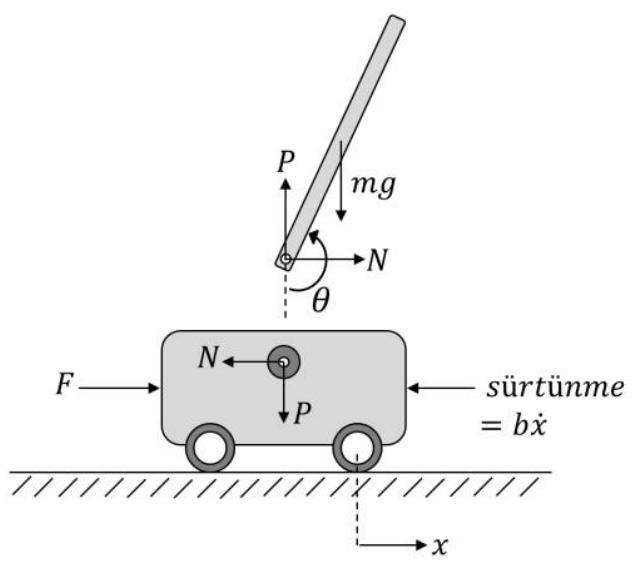

Şekil 2. Ters sarkaç sisteminin serbest cisim diyagramı

Yatay yöndeki arabanın serbest cisim diyagramındaki kuvvetleri toplanarak, aşağıdaki hareket denklemi elde edilmiştir(Control Tutorials for MATLAB and Simulink - Inverted Pendulum: System Modeling, t.y.).

$$
M \ddot{x}+b \dot{x}+N=F
$$

Sarkacın serbest cisim diyagramındaki kuvvetleri yatay yönde toplanarak, reaksiyon kuvveti için aşağıdaki denklem oluş̧urulmuştur.

$$
N=m \ddot{x}+m l \ddot{\theta} \cos \theta-m l \dot{\theta}^{2} \sin \theta
$$

Bu denklem ilk denkleme konulursa, bu sistem için iki yönetim denkleminden biri aşağıda gibi gösterilmiştir.

$$
(M+m) \ddot{x}+b \dot{x}+m l \ddot{\theta} \cos \theta-m l \dot{\theta}^{2} \sin \theta=F
$$


Bu sistem için ikinci hareket denklemini elde etmek için sarkaca dik olan kuvvetler toplanır. Sistemi bu eksen boyunca çözmek matematiği büyük ölçüde basitleştirir. Bunun için aşağıdaki denklem kullanılmıştır.

$$
P \sin \theta+N \cos \theta-m g \sin \theta=m l \ddot{\theta}+m \ddot{x} \cos \theta
$$

Yukarıdaki denklemde P ve N terimlerinden kurtulmak ve aşağıdaki denklemi elde etmek için sarkacın ağırlık merkeziyle ilgili momentler ifade edilmiştir.

$$
-P l \sin \theta-N l \cos \theta=I \ddot{\theta}
$$

Bu son iki ifadeyi birleştirerek, ikinci yönetim denklemi elde edilmiştir.

$$
\left(I+m l^{2}\right) \ddot{\theta}+m g l \sin \theta=-m l \ddot{x} \cos \theta
$$

$\mathrm{Bu}$ çalışmada kullanılacak analiz ve kontrol tasarım teknikleri yalnızca doğrusal sistemler için geçerli olduğundan, bu denklem setinin doğrusallaştırılması gerekmektedir. Spesifik olarak, dikey olarak yukarı doğru denge konumu, $\theta=\pi$ ile ilgili denklemler doğrusallaştırılacak ve sistemin bu dengenin küçük bir yapısı içinde kaldığı varsayılacaktır. Kontrol altında sarkacın dikey olarak yukarı pozisyondan 20 dereceden fazla sapmaması istendiği için bu varsayım makul olarak geçerli olmalıdır. Ø, sarkaç pozisyonunun dengeden sapmasını, yani $\theta=\pi+\emptyset$ ifadesini temsil etsin. Yine dengeden küçük bir sapma ( $\varnothing)$ varsayılırsa, sistem denklemlerinde doğrusal olmayan fonksiyonların aşağıdaki küçük açı yaklaşımları kullanılabilir:

$$
\begin{gathered}
\cos \theta=\cos (\pi+\emptyset) \approx-1 \\
\sin \theta=\sin (\pi+\emptyset) \approx-\emptyset \\
\dot{\theta}^{2}=\dot{\emptyset}^{2} \approx 0
\end{gathered}
$$

Yukarıdaki yaklaşımlar doğrusal olmayan yönetim denklemlerine yerleştirildikten sonra, lineerleştirilmiş iki hareket denklemine ulaşılır. U girişinin F ile değiştirildiğine dikkat edilmesi gerekmektedir.

$$
\begin{aligned}
& \left(I+m l^{2}\right) \ddot{\emptyset}-m g l \varnothing=m l \ddot{x} \\
& (M+m) \ddot{x}+b \dot{x}-m l \ddot{\varnothing}=u
\end{aligned}
$$

Tablo 1. Ters Sarkaç Sistemi Parametreleri

\begin{tabular}{l|c|c}
\hline Sembol & Açıklama & Değer \\
\hline$M$ & Arabanın Kütlesi & $0,5 \mathrm{~kg}$ \\
\hline$m$ & Sarkacın Kütlesi & $0,2 \mathrm{~kg}$ \\
\hline$b$ & Sürtünme Katsayısı & $0,1 \mathrm{~N} /{\mathrm{m} \cdot \mathrm{s}^{-1}}^{-}$ \\
\hline$l$ & Sarkacın Kütle Merkezinden İtibaren Uzaklığı & $0,3 \mathrm{~m}$ \\
\hline$I$ & Sarkacın Atalet Momenti & $0,006 \mathrm{~kg} \cdot \mathrm{m}^{2}$ \\
\hline$F$ & Arabaya Uygulanan Kuvvet & $\mathrm{N}$ \\
\hline$\theta$ & Sarkacın Düşey Eksenle Yaptı̆̆ Açı & Radyan \\
\hline$g$ & Yerçekimi İvmesi & $9,81 \mathrm{~m} / \mathrm{s}^{2}$ \\
\hline
\end{tabular}

Ters sarkaç sisteminin dinamik hareket denklemleri Eşitlik 10 ve 11'de elde edilmiştir. Bu hareket denklemlerine göre LQR kontrolcüsü tasarlanacaktır. LQR kontrolcüsü durum-uzay formunda Eşitlik 12'de gösterildiği modele göre tasarlanacaktır.

$$
\begin{aligned}
& \dot{x}=A x+B u \\
& y=C x+D u
\end{aligned}
$$

Eşitlik 10 ve 11 'de ifade edilen dinamik hareket denklemleri durum-uzay formuna göre modellenme halinde matris gösterimleri Eşitlik 13 ve 14 ’te ifade edilmiştir. 


$$
\begin{aligned}
& {\left[\begin{array}{c}
\dot{x} \\
\ddot{x} \\
\dot{\phi} \\
\ddot{\phi}
\end{array}\right]=\left[\begin{array}{cccc}
0 & 1 & 0 & 0 \\
0 & \frac{-\left(I+m l^{2}\right) b}{I(M+m)+M m l^{2}} & \frac{-\left(I+m l^{2}\right) b}{I(M+m)+M m l^{2}} & 0 \\
0 & 0 & 0 & 1 \\
0 & \frac{-m l b}{I(M+m)+M m l^{2}} & \frac{m g l(M+m)}{I(M+m)+M m l^{2}} & 0
\end{array}\right] \cdot\left[\begin{array}{c}
x \\
\dot{x} \\
\phi \\
\dot{\phi}
\end{array}\right]+\left[\begin{array}{c}
0 \\
\frac{I+m l^{2}}{I(M+m)+M m l^{2}} \\
0 \\
\frac{m l}{I(M+m)+M m l^{2}}
\end{array}\right] \cdot u} \\
& y=\left[\begin{array}{llll}
1 & 0 & 0 & 0 \\
0 & 0 & 1 & 0
\end{array}\right] \cdot\left[\begin{array}{c}
x \\
\dot{x} \\
\phi \\
\dot{\phi}
\end{array}\right]+\left[\begin{array}{l}
0 \\
0
\end{array}\right] \cdot u
\end{aligned}
$$

\subsection{LQR Kontrolcü Tasarımı}

LQR kontrol tekniği çeşitli alanlarda sıklıkla tercih edilen bir kontrol algoritmasıdır. Lineer sistemler için durum-uzay tabanlı tasarlanan bir kontrolcüdür. Herhangi bir sistemin lineer modelinin karesel formda yazıldığında sistemin durumları ile kontrol girişi arasındaki ilişkinin optimize edilmesidir. LQR kontrolcüsü optimize edilmiş kontrol girişini uygulayabilmek için durum değişkenlerini ve maliyet fonksiyonu olan $J$ değerini kullanmaktadır. $J$ değerinin hesaplanıp minimize edilmesiyle optimizasyon işlemi gerçekleştirilmektedir. Ters sarkaç sistemi Eşitlik 12'de belirtildiği şekilde durum-uzay formunda modellenmiştir.

LQR kontrolcüsü tarafında sisteme uygulanacak kontrol girişinin hesaplanmasında kullanılan formüllerden birisi de Eşitlik 15 'te ifade edilen $J$ maliyet fonksiyonudur.

$$
J=\int_{0}^{\infty} x^{T}(t) \cdot Q \cdot x(t)+u^{T}(t) \cdot R \cdot u(t) \cdot d t
$$

Eşitlik $15^{\prime}$ te belirtilen formül içerisinde yer alan $Q$ ve $R$ kazanç matrisleriyle kontrol girişinin tepkileri ayarlanmaktadır. $Q$ kazanç matrisiyle kontrol işleminin hassasiyeti, $R$ kazanç matrisiyle sistem girişleri veya bozucu etkilere karşı verilen agresiflik ayarlanmaktadır. LQR kazanç vektörü aşağıdaki gibi ifade edilmektedir:

$$
K=R^{-1} B^{T} P
$$

Eşitlik 16'da belirtilen $P$ değeri, Eşitlik 17'de cebirsel Ricatti denkleminin çözümünden elde edilen matrisdir.

$$
A^{T} P+A P-P B R^{-1} B^{T} P+Q=0
$$

LQR kontrolcü parametrelerini belirmede genellikle deneme yanılma yöntemi kullanılmaktadır. Bu yöntem kullanıcının zamanını aldığ 1 gibi belirlenen parametlerin en iyi olduğu konusunda da bir tespiti bulunmamaktadır. Parametrelerin ayarlanmasında hem zaman almayacak hemde en iyiyi arama yaklaşımı içinde olan optmizasyon algoritmaları burada devreye girmektedir. Optimizasyon algoritmaları hesaplama yükünü artırmasına rağmen en iyi parametre değerinin bulunması garanti etmektedir.

\subsection{Genetik Algoritma}

GA optimizasyon probleminin en uygun sonucunu bulmak için çalışan bir arama algoritmasıdır. John Holland tarafindan 1975 'te önerilmiştir. GA temel olarak doğal seçilim, mutasyon ve çaprazlama operatörlerinden oluşmaktadır. GA popülasyon tabanlı bir arama algoritması olup belirlenen tasarım kriterini opimize edecek sonuçlar bulmayı hedeflemektedir. Popülasyon içerisindeki güçlü bireyleri çaprazlama işlemiyle bir sonraki nesile aktarma yaklaşımıyla en iyi sonuca ulaşabilmektedir. GA şematik akış diyagramı Şekil 3’te gösterilmiştir. 


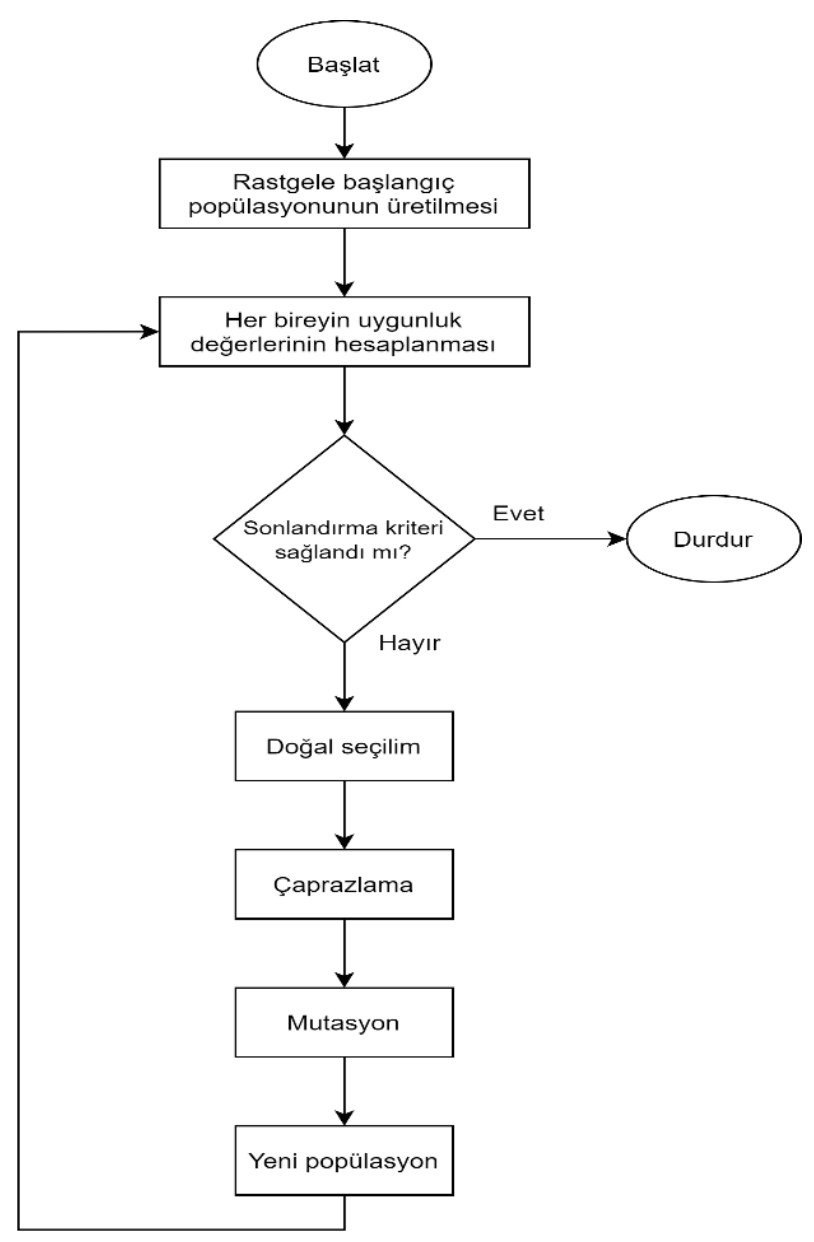

Şekil 3. Genetik algoritma akış diyagramı

\section{Araştırma Sonuçları ve Tartışma}

LQR kontrolcüsünün kazanç matrislerini deneme yanılma yöntemiyle bulunmasındaki zorluklardan ve kaybedilen zamandan bahsedilmiştir. $\mathrm{Bu}$ durumun önüne geçmek için optimizasyon algoritmalarıyla LQR kontrolcüsünün parametreleri için en iyiyi bulmaya yönelik çeşitli çalışmalar mevcuttur (Ata \& Çoban, 2017; Önen vd., t.y.). Bu çalışmada deneme yanılma yöntemiyle bulunan LQR kontrolcü parametreleri (Control Tutorials for MATLAB and Simulink - Inverted Pendulum: System Modeling, t.y.) ile GA sayesinde tespit edilen parametreler karşılaştırılacaktır. Ayrıca GA uygunluk fonksiyonunda farklı olarak kullanılan formülasyondan bahsedilecektir.

GA, Parçacık Sürü Optimizasyonu (PSO) ve Yapay Arı Kolonisi (ABC) gibi optimizasyon algoritmalarıyla LQR kontrolcüsünün $Q$ ve $R$ parametreleri optimize edilirken uygunluk fonksiyonlar genellikle referans sinyal değerinin; yükselme zamanı, oturma zamanı ve kalıcı durum hatası üzerinden işlem yapılmaktadır (Ata \& Çoban, 2017; Önen vd., t.y.). Bu çalışmada farklı olarak kullanılan uygunluk fonksiyonu Eşitlik 18'de belirtilmiştir.

$$
\begin{gathered}
f_{\text {uygunluk }}=\text { referans } \text { sinyali }-x_{r m s} \\
x_{r m s}=\sqrt{\frac{1}{n} \sum_{i=1}^{n} x_{i}^{2}}
\end{gathered}
$$

Eşitlik 18 'de uygunluk fonksiyonu, ters sarkaç sistemine referans olarak verilen arabanın konumu ve kazanç matrisleri belirlenmiş kontrolcü girişinin uygulanması sonucunda arabanın mevcut konumunun kare ortalamalarının karekök değeri alınarak elde edilen sonucun birbirinden çıkartılmasına dayanmaktadır. Deneme yanılma yöntemiyle tespit edilen LQR kontrolcü parametreleri Eşitlik 20'de gösterilmiştir. $Q$ ve $R$ parametleri sonucunda elde edilen $K$ geri besleme kazanç matrisi de aşağıda gösterilmiştir. 


$$
\begin{gathered}
Q_{\operatorname{man}}=\left[\begin{array}{cccc}
5000 & 0 & 0 & 0 \\
0 & 0 & 0 & 0 \\
0 & 0 & 100 & 0 \\
0 & 0 & 0 & 0
\end{array}\right] \\
R_{\text {man }}=[1] \\
K_{\text {man }}=\left[\begin{array}{llll}
-70.7107 & -37.8345 & 105.5298 & 20.9238
\end{array}\right]
\end{gathered}
$$

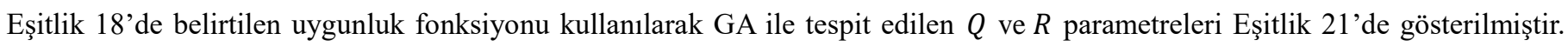
Tespit edilen kontrolcü parametreleriyle hesaplanan $K$ geri besleme kazan matrisi de aynı eşitlikte ifade edilmiştir.

$$
\begin{gathered}
Q_{G A}=1 e 3 .\left[\begin{array}{cccc}
7.2924 & 0 & 0 & 0 \\
0 & 0 & 0 & 0 \\
0 & 0 & 0.1306 & 0 \\
0 & 0 & 0 & 0
\end{array}\right] \\
R_{G A}=[0.059] \\
K_{G A}=[-109.7086-55.8749145 .8017 \text { 29.0067 }]
\end{gathered}
$$

Deneme yanılma yöntemiyle ve GA ile tespit edilen $K$ geri besleme kazanç matrisini karşılaştırmak Matlab/Simulink modeli tasarlanmıştır. LQR kontrolcüsüyle sarkacın açısını dengede tutmak hedeflerden birisidir. Diğer bir hedef ise arabayı da istenilen referans konuma gönderilmesini sağlamaktır. Kurulan Simulink modelinde ters sarkaç sisteminde araba 0,2 metre ileriye gidecek şekilde referans sinyali uygulanmaktadır. Verilen referans değerine karşı standart LQR ve GA ile optimize edilmiş LQR kontrolcüsünün karşılaştırmaları Şekil 4 'te gösterilmiştir.

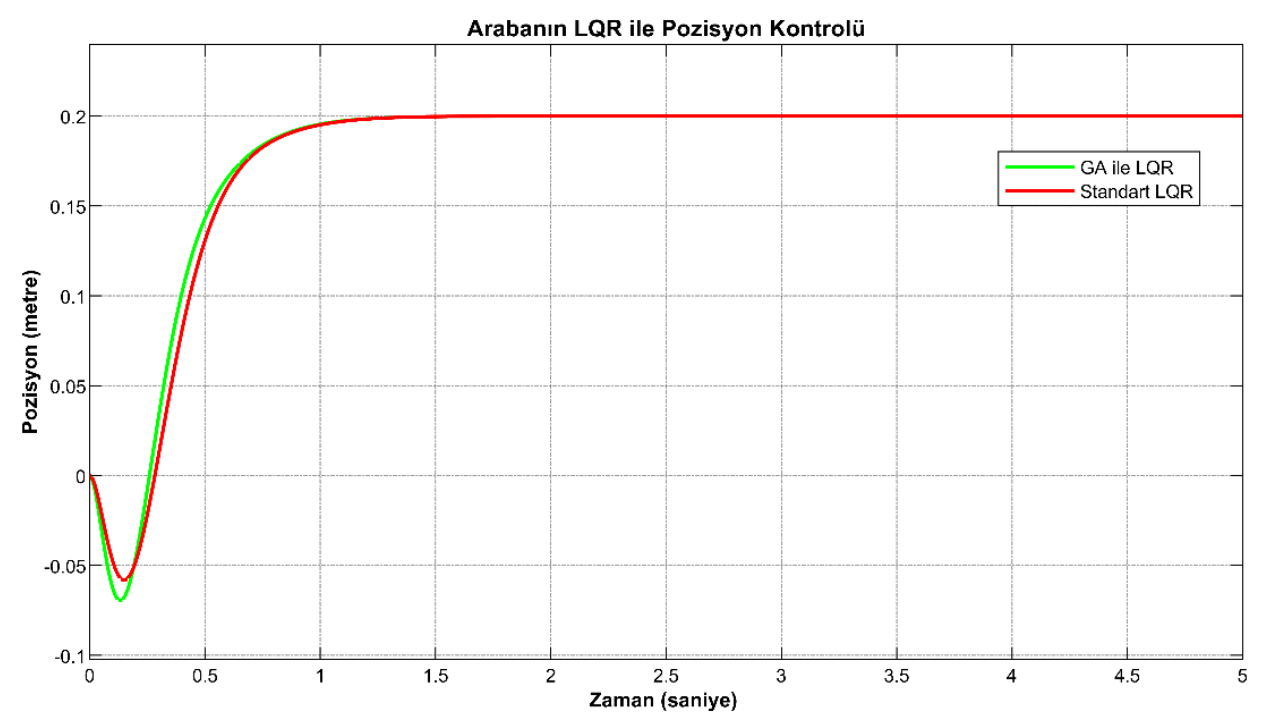

Şekil 4. LQR kontrolcü ile pozisyon hedefine ulaşma durumlarının karşılaştırılması

Referans sinyale yükselme süreleri karşılaştırıldığında standart LQR kontrocüsü 0,412 saniyede ulaşırken, GA ile optimize edilmiş LQR kontrolcüsü 0,424 saniyede ulaşmıştır. Referans sinyale oturma süreleri karşılaştırıldığında standart LQR kontrolcüsü 0,988 saniyede ulaşırken, GA ile optimize edilmiş LQR kontrolcüsü 0,965 saniyede ulaştığı görülmüştür. Yükselme ve oturma zamanları toplandığında GA ile optimize edilmiş LQR kontrolcüsünün daha başarılı olduğu gözlemlenmiştir.

LQR kontrolcüsünün iki tasarım kriteri olduğu daha öncesinde belirtilmiştir. Simulink modelinde 0,2 metre referans sinyali uygulanırken ters sarkaçta dengelenmiş durumda olması gerekmektedir. Belirlenen referans pozisyon bilgisine ulaşırken aynı zamanda sarkacında dengelenmesi gerekmektedir. Sarkacın aldığı açı değerleri Şekil 5 'te gösterilmiştir. 


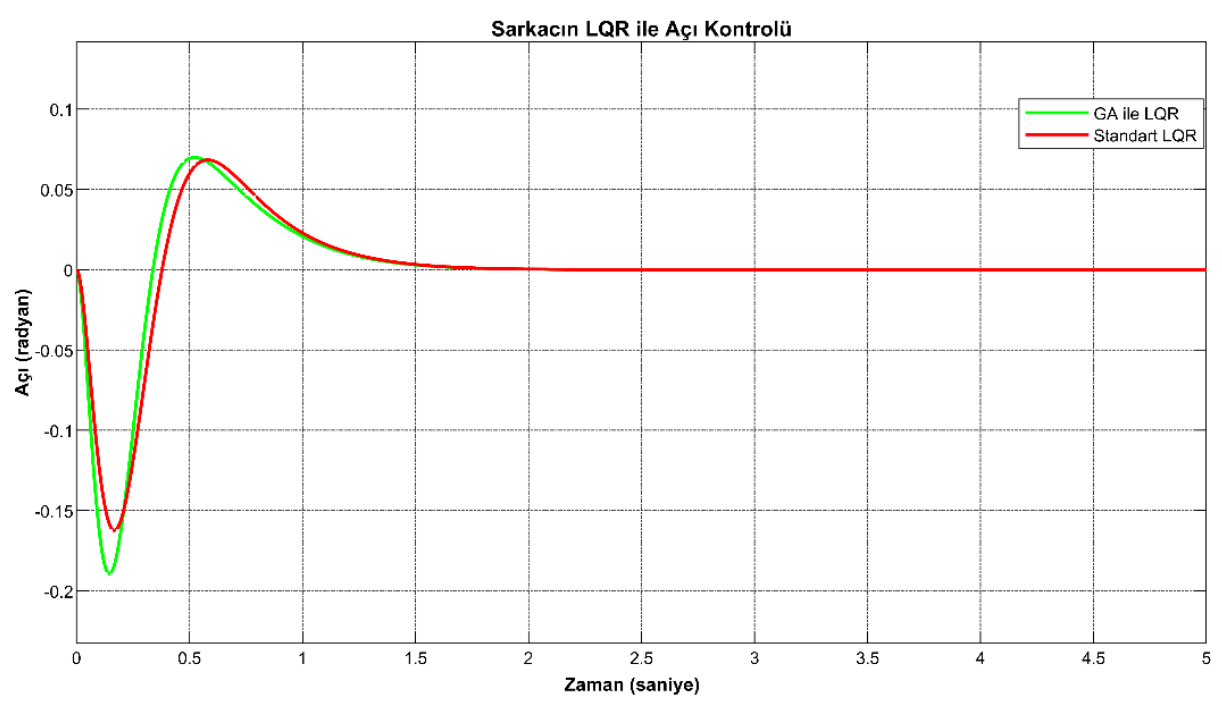

Şekil 5. LQR kontrolcü ile sarkacın dengelenmesinin karşılaştırılması

Sarkacın dengelenmesindeki performansları oturma sürelerine göre karşılaştıııldığında standart LQR kontrolcüsü 1,501 saniyede ulaşırken, GA ile optimize edilmiş LQR kontrolcüsü 1,441 saniyede ulaştı̆̆ı görülmüştür. Sarkacın dengelenmesinde oturma zamanları incelendiğinde GA ile optimize edilmiş LQR kontrolcüsünün daha başarılı olduğu gözlemlenmiştir.

Ters sarkacın belirlenen pozisyon değerine ulaşabilmesi için bir kontrol girişi uygulanması gerekmektedir. LQR kontrolcünün üretmiş olduğu kontrol sinyal girişlerinin karşılaştırması Şekil 6'da gösterilmiştir.

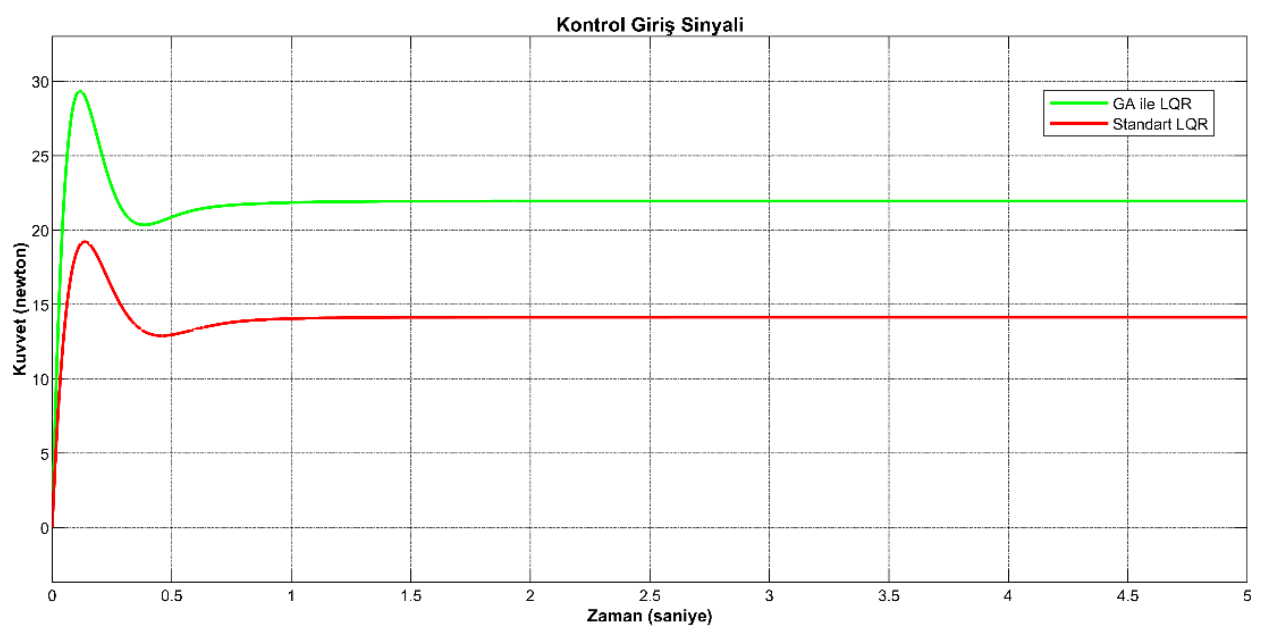

Şekil 6. LQR kontrolcü tarafindan ters sarkaca uygulanan kontrol girişleri

Ters sarkacın verilen referans pozisyonda tutulması için uygulanması gereken kontrol sinyal girişleri incelendiğinde GA ile optimize edilmiş kontrolcünün 21,941 N kuvvete ihtiyacı olurken, standart LQR kontrolcüsünün 14,142 N kuvvete ihtiyacı olduğu gözlemlenmiştir. Ters sarkaç sisteminin deneysel olarak gerçeklenmesi durumunda tasarım parametrelerine göre ortaya çıkan kuvvet değerinin göz önüne alınması için kontrol sinyal girişinin değerleri gösterilmiştir.

\section{Sonuç}

$\mathrm{Bu}$ çalışmada LQR kontrol algoritması parametreleri GA ile belirlenmiştir. GA uygunluk fonksiyonu olarak mevcut çalışmalarda kullanılan referans sinyale yükselme süresi, oturma süresi ve kalıcı durum hatası gibi değerlendirme kriterlerinin aksine farklı bir yaklaşım önerilmiştir. Önerilen yaklaşım ile tespit edilen LQR kontrolcü parametreleri, deneme yanılma yöntemiyle tespit edilen parametrelere göre daha başarılı olmuştur. Kontrolcü parametrelerinin deneme yanılma yöntemiyle bulunmasında kaybedilen zamanında önüne geçilmiş̧ir.

Simulink ortamında modellenen ters sarkaç sistemi ileride yapılacak çalışmalarda GA, PSO ve ABC gibi optimizasyon algoritmalarıyla kontrolcü parametrelerinin tespit edilmesi ve performanslarının karşılaştırılması planlanmaktadır. Aynı zamanda Simulink ortamında modellenen sistemin deney düzeneği kurularak kontrolcü performansının uygulama üzerinde gözlemlenmesi hedeflenmektedir. LQR kontrolcü parametrelerinin optimizasyon algoritmalarılla belirlenmesinin haricinde farklı kontrol teknikleri ile de karşılaştırılması ileride yapılaca çalışmalar arasındadır. Özellikle ters sarkaç parametre değişimlerine ve belirsizliklerine karşı gürbüz kontrol tekniğinin uygulanması düşünülmektedir. 


\section{Kaynakça}

Anderson, C. W. (1989). Learning to Control and Inverted Pendulum Using Neural Networks. IEEE Control Systems Magazine, 9(3), 31-37. https://doi.org/10.1109/37.24809

Ata, B., \& Çoban, R. (2017). Linear Quadratic Optimal Control of an Inverted Pendulum on a Cart Using Artificial Bee Colony Algorithm: An Experimental Study. 16.

Bakarac, P., Klauco, M., \& Fikar, M. (2018). Comparison of Inverted Pendulum Stabilization with PID, LQ, and MPC Control. 2018 Cybernetics \& Informatics (K\&I), 1-6. https://doi.org/10.1109/CYBERI.2018.8337540

Bilgiç, H. H., Şen, M. A., Kalyoncu, M., \& Yapıcı, A. (2014). Doğrusal Ters Sarkacın Denge Kontrolü için Yapay Sinir Ă̆ı Tabanlı Bulanık Mantık \& LQR Kontrolcü Tasarımı. https://doi.org/10.13140/RG.2.1.4983.7204

Control Tutorials for MATLAB and Simulink-Inverted Pendulum: System Modeling. (t.y.). Geliş tarihi 09 Ekim 2020, gönderen http://ctms.engin.umich.edu/CTMS/index.php?example=InvertedPendulum\&section=SystemModeling

Ghanbari, A., \& Farrokhi, M. (2006). Decentralized Neuro-Fuzzy Controller Design Using Decoupled Sliding-Mode Structure for Two-Dimensional Inverted Pendulum. 2006 IEEE International Conference on Engineering of Intelligent Systems, 1-6. https://doi.org/10.1109/ICEIS.2006.1703155

Housner, G. W. (1963). The Behavior of Inverted Pendulum Structures During Earthquakes. Bulletin of the Seismological Society of America, 53(2), 403-417.

Mahfouz, A. A., M. K., M., \& Salem, F. A. (2013). Modeling, Simulation and Dynamics Analysis Issues of Electric Motor, for Mechatronics Applications, Using Different Approaches and Verification by MATLAB/Simulink. International Journal of Intelligent Systems and Applications, 5(5), 39-57. https://doi.org/10.5815/ijisa.2013.05.06

Muskinja, N., \& Tovornik, B. (2006). Swinging Up and Stabilization of a Real Inverted Pendulum. Industrial Electronics, IEEE Transactions on, 53, 631-639. https://doi.org/10.1109/TIE.2006.870667

Okubanjo, A. A., \& Oyetola, O. K. (2019). Dynamic Mathematical Modeling and Control Algorithms Design of an Inverted Pendulum System (IPS). Turkish Journal of Engineering, 1-10. https://doi.org/10.31127/tuje.435028

Önen, Ü., Çakan, A., \& İLhan, İ. (t.y.). Performance comparison of optimization algorithms in LQR controller design for a nonlinear system. 16.

Prasad, L., Tyagi, B., \& Gupta, H. (2014). Optimal Control of Nonlinear Inverted Pendulum System Using PID Controller and LQR: Performance Analysis Without and With Disturbance Input. International Journal of Automation and Computing, 11, 661-670. https://doi.org/10.1007/s11633-014-0818-1

Razzaghi, K., \& Jalali, A. A. (2012). A New Approach on Stabilization Control of an Inverted Pendulum, Using PID Controller. Advanced Materials Research, 4674-4680. https://doi.org/10.4028/www.scientific.net/AMR.403-408.4674

Shehu, M., Ahmad, M. R., Shehu, A., \& Alhassan, A. (2015). LQR, Double-PID and Pole Placement Stabilization and Tracking Control of Single Link Inverted Pendulum. 2015 IEEE International Conference on Control System, Computing and Engineering (ICCSCE), 218-223. https://doi.org/10.1109/ICCSCE.2015.7482187

Wang, J.-J. (2011). Simulation Studies of Inverted Pendulum Based on PID Controllers. Simulation Modelling Practice and Theory, 19(1), 440-449. https://doi.org/10.1016/j.simpat.2010.08.003

Yeung, K. S., \& Chen, Y. P. (1990). Sliding Mode Controller Design of a Single-Link Flexible Manipulator Under Gravity. International Journal of Control, 52(1), 101-117. https://doi.org/10.1080/00207179008953526 